\title{
Research on the synthesis of chitosan@MIL-100(Fe)
}

\author{
SONG Zurong ${ }^{1}$, TAO Ali ${ }^{1}$, FENG Xuehua ${ }^{1}$ \\ ${ }^{1}$ College of Pharmacy, Anhui Xinhua University, Anhui, Hefei, 230088
}

\begin{abstract}
Objective: Studying new carrier materials to solve the problems of bioavailability and side effects of drugs in the body and choose the suitable carrier to coat the drug. This carrier not only improves the release and absorption of drug molecules in the body, but also significantly improves the bioavailability and reduces the toxic side effects of high concentrated drugs. Therefore, this paper focuses on the research of the synthesis of a new carrier material.

Methods: Using ferric nitrate as iron source, first add ferric nitrate, 1,3,5-benzene tricarbonic acid, chitosan solution and deionized water pro rata, then synthesize the product using mechanical stirring, and purify and dry the product.

Results: The maximum yield of chitosan is obtained when the concentration of chitosan is $1 \%$ using mechanical stirring method, and the yield is $0.6330 \mathrm{~g}$.

Conclusion: The results show that the thinner the concentration of chitosan solution is, the better synthesizes the chitosan@MIL-100(Fe) and the higher the yield is. And it reaches the best result when the concentration is $1 \%$.
\end{abstract}

\section{Introduction}

There is a storm of researches on drug sustained and controlled release systems at home and abroad nowadays. The preparation that controls or delays drug release and sustains the release is sustained and controlled release preparations in the drug sustained and controlled release system. We are very optimistic about its development as a new drug dosage form in future. Therefore, it's important to deeply explore how to obtain new carrier materials with smaller doses, lower toxic and side effects, and better curative effects to improve the sustained and controlled release system of drugs and help to improve the bioavailability of drugs in the body. Sodium alginate, water-soluble polyvinyl alcohol, magnetic attapulgite, modified chitosan, MOFs and other materials can be used as carrier materials for pharmaceutical preparations, among which chitosan with its good biocompatibility and easy degradability and MIL-100(Fe) with its high drug loading rate and a certain sustained release effect are being used in drug carriers. And combining the two components will give full play to their respective advantages to achieve complementary effect, which not only realizes high drug loading rate but also solves the internal rejection problem to promote drug absorption, and thus improve the bioavailability. On the other hand, it's simple and easy to operate the synthesis process of chitosan@MIL-100(Fe) ${ }^{[1,2]}$. Therefore, as a new type of drug carrier material, chitosan@MIL-100(Fe) is proved to be successful and the research at home and abroad is gradually developed towards chitosan polymer materials and the grafting of chitosan with different substances as drug carriers has gradually been brought into focus.

There are not many research reports on chitosan @MIL-100(Fe) at home and abroad yet, but the current research on improving the biocompatibility of MOFs proves to be a good start. And studies show that chitosan has good biodegradability, biocompatibility, non-immunogenicity, mucosal adsorption property and the ability to improve absorption and controls drug release ${ }^{[3,4]}$. In addition, when chitosan is used as drug carrier, it stabilizes or protects the active ingredients of the drug, promotes the absorption, delays or controls the release of the drug, and helps to deliver the drug to the target organs and tissues to play its unique role ${ }^{[5]}$. Therefore, the new carrier with chitosan as the coating material of MIL-100 (Fe) is more conducive to improve the pharmacological efficiency and the drug utilization and also features in high drug loading rate, target targeting, and advantages like good modifiable affinity, non-toxicity, good biocompatibility and easy degradability. It not only compensates for the biocompatibility problem of MIL-100(Fe), but also improves the pharmacological toxicity of the drug-chitosan@MIL-100(Fe) compound particles. If the safety problem of the carrier material application is solved, there will be better development of the new drug delivery system and the drug also effects better, thereby it helps to improve the prevention and treatment of diseases $^{[6]}$. Therefore, the new carrier material synthesized in this article has its application value. 


\section{Experimental materials and methods}

\subsection{Materials and instruments}

Chitosan, 1,3,5-benzene tricarbonic acid, N-N-dimethyl formamide (Shanghai Aladdin Bio-Chem Technology Co., Ltd); ferric nitrate, $\mathrm{FeCl}_{3} \cdot 6 \mathrm{H} 2 \mathrm{O}$, acetic acid, ethanol (Tianjin Guanfu Fine Chemical Research Institute); DF-101 Z collective heating stirrer DF-101 Z (Changzhou Putian Instrument Manufacturing Co., Ltd); circulating water vacuum pump SHZ-D(III) (Gongyi Yuhua Instrument Co., Ltd); electric thermostatic blast drying oven DHG-9101-SOA (Shanghai Sanfa Scientific Instruments Co., Ltd); transmission scanning electron microscope Nano SEM450 (Qinhuangdao Taijihuan Nano Product Co., Ltd).

\subsection{Methods}

\subsubsection{Preparation of chitosan solution}

(1) The first group: dissolve chitosan $(0.3 \mathrm{~g})$ in $30 \mathrm{ml}$ of acetic acid $[2.0(\mathrm{v} / \mathrm{v})]$ aqueous solution, and stir with ultrasonic at room temperature for $1.5 \mathrm{~h}$ to prepare chitosan solution $[1 \%(\mathrm{w} / \mathrm{v})]$.

(2) The second group: dissolve chitosan (0.3 g) in 20 $\mathrm{ml}$ of acetic acid $[2.0(\mathrm{v} / \mathrm{v})]$ aqueous solution, and stir with ultrasonic at room temperature for $1.5 \mathrm{~h}$ to prepare chitosan solution $[1.5 \%(\mathrm{w} / \mathrm{v})]$.

(3) The third group: dissolve chitosan $(0.3 \mathrm{~g})$ in $10 \mathrm{ml}$ of acetic acid $[2.0(\mathrm{v} / \mathrm{v})]$ aqueous solution, and stir with ultrasonic at room temperature for $1.5 \mathrm{~h}$ to prepare chitosan solution $[3 \%(\mathrm{w} / \mathrm{v})]$.

\subsubsection{Preparation of chitosan@MIL-100(Fe)}

(1) Ferric nitrate as iron resource

In the ratio of $1.0 \mathrm{Fe}\left(\mathrm{NO}_{3}\right)_{3:} 0.661,3,5-\mathrm{BTC}: 35 \mathrm{H} 2 \mathrm{O}$

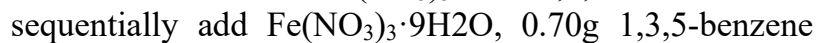
tricarbonic acid and $3 \mathrm{ml}$ deionized water into the round bottom flask, add $10 \mathrm{ml}$ of chitosan solution, and magnetically stir for about $30 \mathrm{~min}$, condense and reflux, then adjust the temperature to $95^{\circ}$ and keep it for $12 \mathrm{~h}$. After the end of the reaction, wash, filter and dry the crude product ${ }^{[7]}$.

According to the above experimental process to prepare 3 copies of products with the chitosan concentration of $1 \%, 1.5 \%$, and $3 \%$ respectively. After drying, wash and purify the product and add $1.0 \mathrm{~g}$ of the product into a round bottom flask with $200 \mathrm{ml}$ of deionized water. After stirring for 5 hours at a temperature of $80^{\circ}$, wash and dry the product, then add $50 \mathrm{ml}$ of absolute ethanol, and stir at a constant temperature of $60^{\circ}$ for 3 hours. After the end of the reaction, vacuum filter the product and wash with $\mathrm{N}-\mathrm{N}$-dimethyl formamide, ethanol and deionized water in sequence until the mother liquor is colorless and transparent, and finally dry in a drying oven to get the orange powder (see Figure 2 in the Appendix).

(1) Ferric chloride as iron resource (comparative experiment)

In the ratio of $1.0 \mathrm{FeCl}_{3:} 0.67$ 1,3,5-BTC:35 H2O sequentially add $1.35 \mathrm{~g} \mathrm{FeCl}_{3} \cdot 6 \mathrm{H} 2 \mathrm{O}, 0.70 \mathrm{~g} \mathrm{1,3,5- \text {benzene }}$ tricarbonic acid and $3 \mathrm{ml}$ deionized water into the round bottom flask, add $10 \mathrm{ml}$ of $1 \%$ chitosan solution, and magnetically stir for about $30 \mathrm{~min}$, condense and reflux, then adjust the temperature to $95^{\circ}$ and keep it for $12 \mathrm{~h}^{[8]}$. After the end of the reaction, wash and dry the product.

Wash and purify the product after drying it in the same way as with the ferric nitrate method.

\subsubsection{Synthesis method of chitosan@MIL-100(Fe)}

Method 1: prepare the chitosan solution first, synthesize MIL-100(Fe) and cross-link the two in $25 \%$ glutaraldehyde, then heat the mixed solution in a water bath at $40^{\circ} \mathrm{C}$ and stir continuously for 90 minutes. Next step is to adjust the $\mathrm{pH}$ of the solution to 9 to 10 with 1 $\mathrm{ml} \mathrm{NaOH}$, and heat it in a water bath at $70^{\circ} \mathrm{C}$ for 60 minutes to get the product. Wash the final product with $\mathrm{N}-\mathrm{N}$-dimethyl formamide, ethanol and deionized water three times.

Method 2: Add chitosan solution to the raw materials of MIL-100 (Fe). The experimental method is the same as synthesizing MIL-100 (Fe). Wash the purified product respectively with $\mathrm{N}-\mathrm{N}$-dimethyl formamide, ethanol and deionized water three times. This method is suitable for synthesizing MIL-100 (Fe) at low temperature, because chitosan is not resistant to high temperature.

\section{Results and analysis}

\section{1 morphology characterization of chitosan@MIL-100(Fe)}

Adjust the crystal size of chitosan@MIL-100(Fe) from $200 \mathrm{~nm}$ to $0.2 \mathrm{um}$. Analyze the crystal morphology of the material to analyze the crystal structure of chitosan@MIL-100(Fe) with a scanning electron microscope (SEM). See Figurel and Figure 2: 


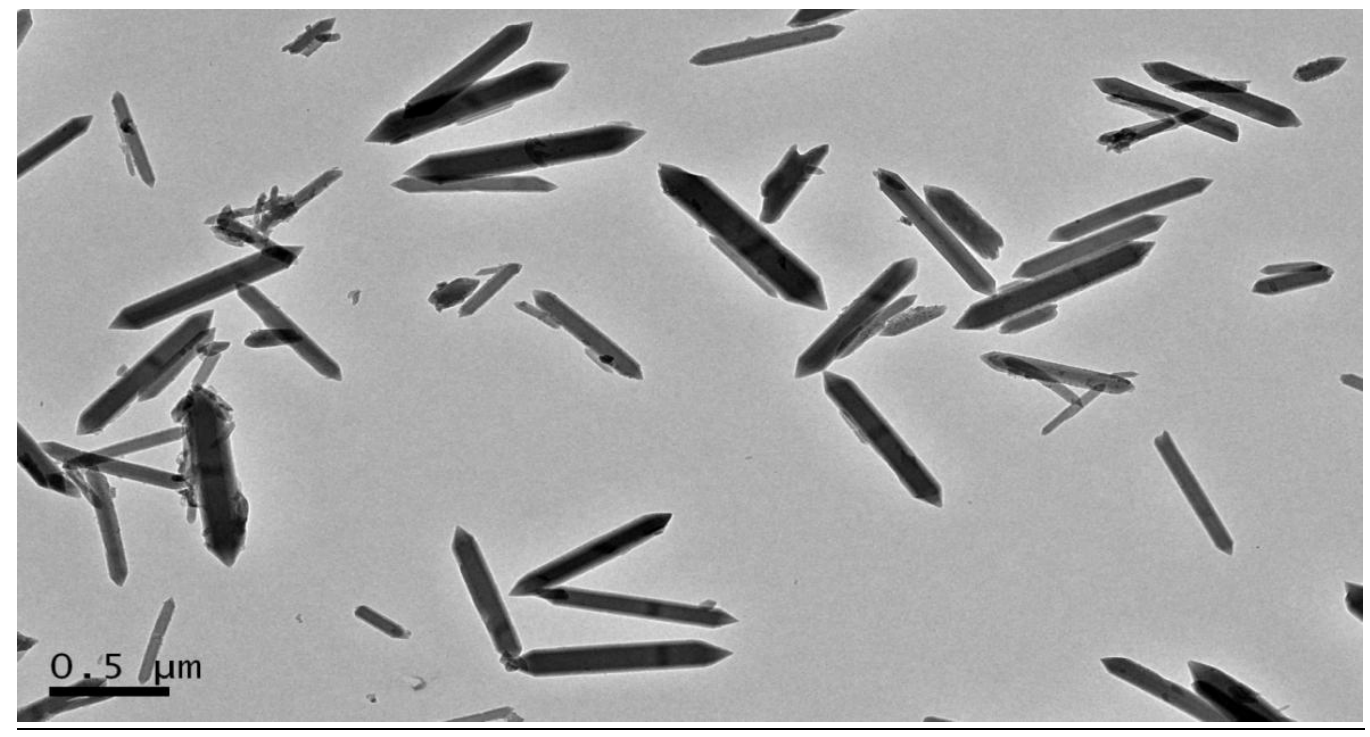

Fig. 1 Picture of chitosan@MIL-100(Fe) under 0.5 micrometer high resolution transmission electron microscope

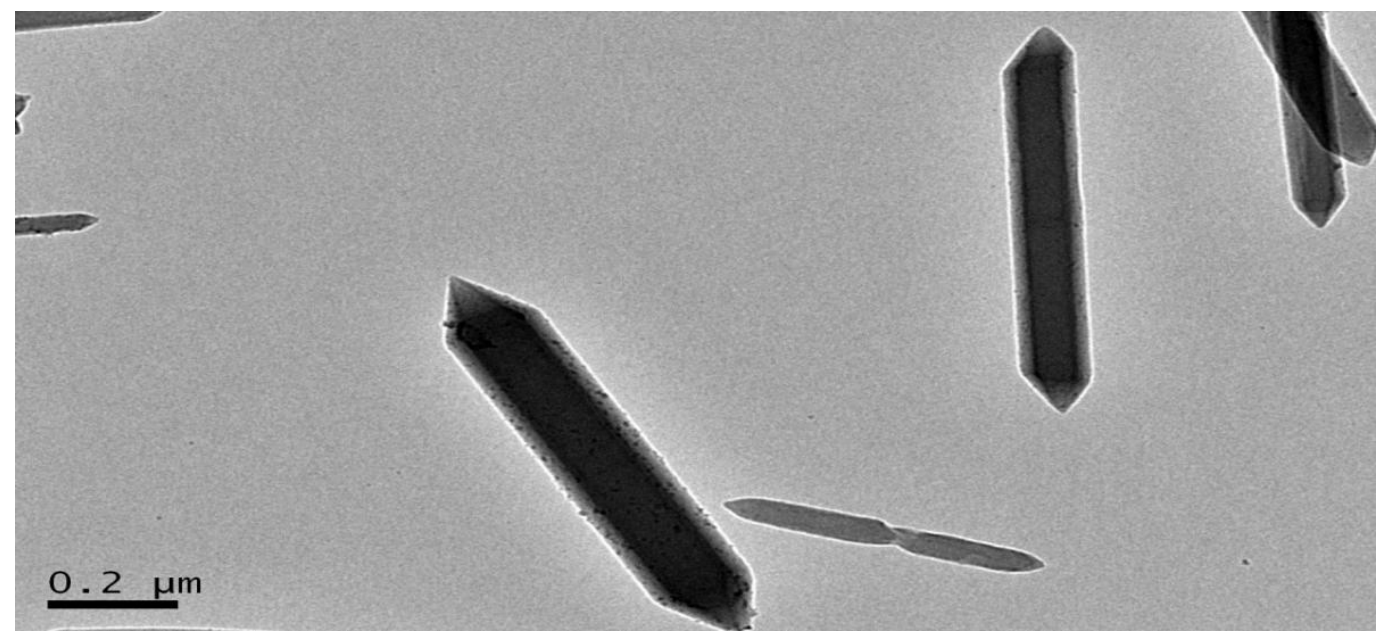

Fig. 2 Picture of chitosan@MIL-100(Fe) under 0.2 micrometer high resolution transmission electron microscope

\subsection{Experimental results of chitosan@MIL-100(Fe)}

(1) Ferric nitrate as iron resource

$1 \%$ chitosan produces $0.6330 \mathrm{~g}$ pure product, $1.5 \%$ chitosan produces $0.6317 \mathrm{~g}$ pure product and $2 \%$ chitosan produces $0.5832 \mathrm{~g}$ pure product. Which tells that the highest yield can be obtained when the concentration of chitosan is $1 \%$.

(2) Ferric chloride as iron resource

$1 \%$ chitosan produces $0.3529 \mathrm{~g}$ pure product.

\section{Conclusions}

The shape of the finished product is not much different from pure MIL-100 (Fe) under the scanning electron microscope. The difference is that the finished product has a layer of protective film (chitosan) on the shell which the pure MIL-100 (Fe) (see Figure3 and Figure 4) doesn't have. Therefore. It can be concluded that the chitosan solution coats MIL-00(Fe) uniformly, and the finished product is indeed chitosan@MIL-100(Fe). 


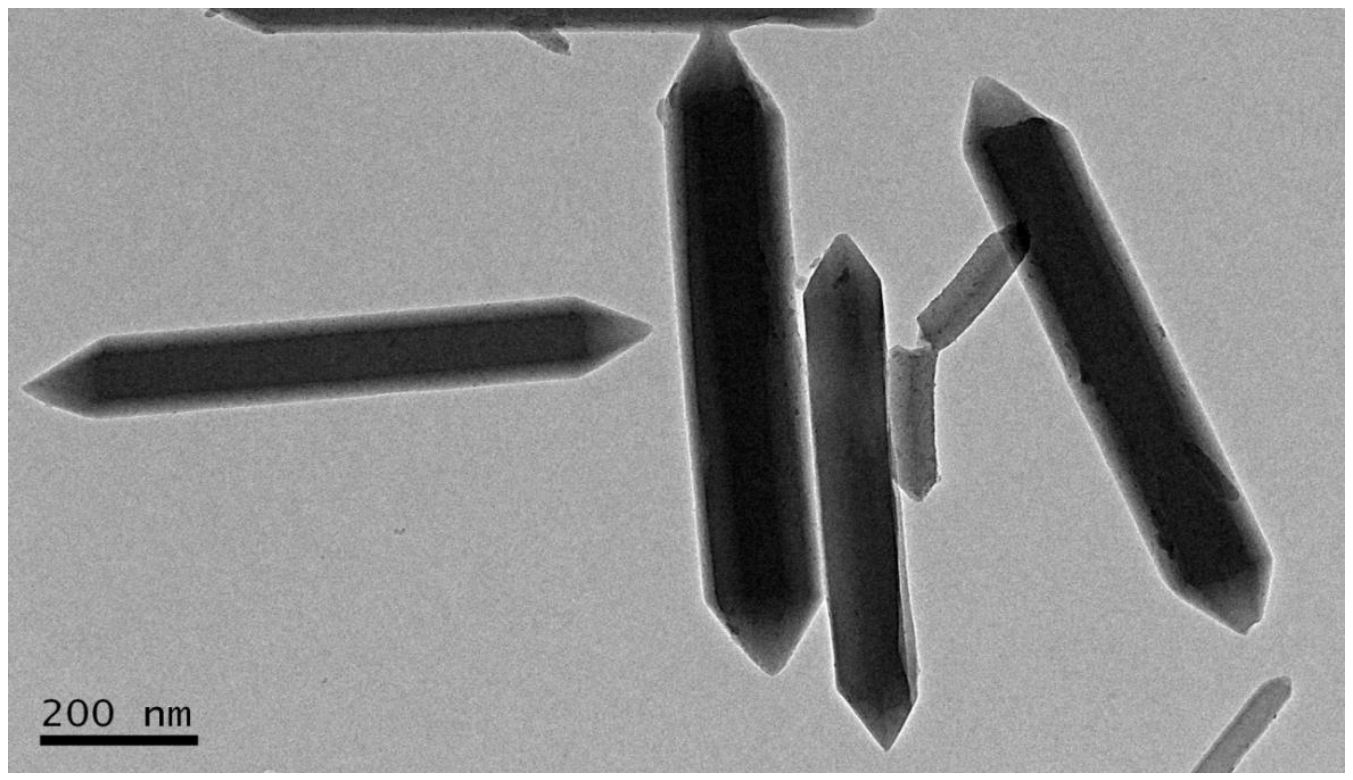

Fig. 3 Picture of pure MIL-100(Fe) under 200nm transmission electron microscope

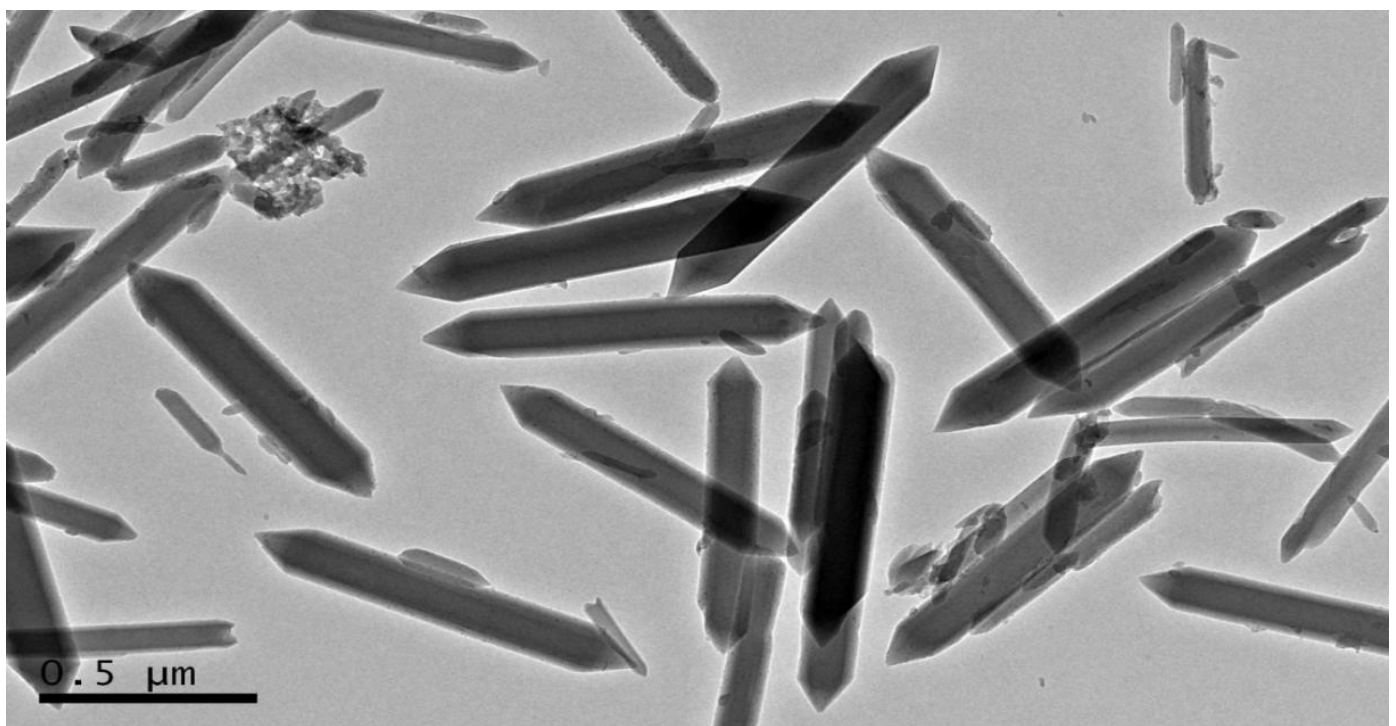

Fig. 4 Picture of pure MIL-100(Fe) under 0.5um transmission electron microscope

The experimental results show that the lower the concentration of the chitosan solution is, the higher the yield of the pure product is. That is to say, the lower concentration of chitosan benefits the synthesis of the product. A set of comparative experiments was conducted on this basis and the results show that the yield from the experiment with ferric nitrate as the iron source is much more than that from the one with ferric chloride as the iron source. So it can be concluded that using ferric nitrate as the iron source is more conducive to the synthesis of the product than using ferric chloride as the iron source.

\section{Acknowledgements}

This work was supported by Natural Science Research Foundation of the Department of Education of Anhui Province (No.KJ2018A0583 , KJ2019A0874 , KJ2020A0789),
Scientific research team of Anui Xinhuauniversity (kytd201908 ),

Quality engineering project of Anhui Xinhua University（2019xqjdx03，2019jxjy45）,

University level scientific research project of Anhui Xinhua University (2017zr010).

\section{References:}

1. Mladenovska. K,CruawJ.O,Richomme.P.,et al. Goracinovh K. 5-ASA loadd chnosan CaaJgi-natt miavpardcla: prcparation and physicochc;mical damrizahentI. Imtmational JoumaJ of Phamacadcs 2007.345:5949.

2. Hillaireau H,Couvreur P.Nanoencapsulation of antiviralnucleotide analogs[J]. Journal of Dr-ug Delivery Scienceand Technology, 2009, 19(6): 385-390. 
3. Ma Aihua, Hu Tingwei, Jia Qingming, et al. Metal-organic frameworks used as controlled release carriers for various drugs[J].Kunming University of Science and Technology,2016,3(47):03033-03034..

4. Zhai Rui, Jiao Fenglong, Lin Hongjun, et al. Research progress of metal organic framework materials[J].Chromatography.2014,2,32(2):113.

5. Cao Wenjiang, Cheng Jiang, Wang Changguang. Research progress of carrier materials for nano-targeted drug delivery systems $[\mathrm{J}]$. Chinese Tissue Engineering Research and Clinical Rehabilitation,2007,11(22):4380-4383.

6. Wang Yiting, Chen Yong, Hong Yan. The clinical application prospect of chitosan as drug carrier and gene carrier[J]. Chinese Tissue Engineering Research and Clinical Rehabilitation, 2008.12(10): 1895.

7. Wang Zhiqiang, Fan Yuling, Meng Xiangyu, et al. Research progress of chitosan as drug carrier[J]. Science and Technology Forum 2014 (92): 54-55.

8. Huo Meifu, Zhou Jianping, Wei Yan, et al. Preparation of paclitaxel anion chitosan micelles and study on tissue distribution in mice[J]. Chinese Pharmaceutical Journal, 2006, 41(24): 1876-1881. 